\title{
Intensidade e Dinâmica da Modernização Agrícola no Brasil e nas Unidades da Federação*
}

\author{
Paulo Marcelo de Souza ${ }^{* *}$ \\ João Eustáquio de Lima***
}

Sumário: 1. Introdução; 2. Objetivos; 3. Metodologia; 4. Resultados e discussão; 5. Conclusões.

Palavras-chave: modernização agrícola; análise fatorial; tecnologia

Códigos JEL: Q10; Q16; Q18.

Este artigo apresenta um estudo cujo objetivo foi caracterizar a evolução do processo de modernização agrícola nas unidades da Federação brasileira, utilizando a análise fatorial aplicada a um conjunto de variáveis relacionadas ao emprego das principais tecnologias modernas. Os resultados permitiram concluir que a intensidade do processo de modernização foi significativamente diferente entre as unidades da Federação. Esse processo sofreu retração a partir de 1980, com a redução nos valores associados ao nível de financiamento e investimentos no setor, fenômeno relacionado à redução na oferta de crédito rural e à elevação de seu custo, e possivelmente influenciado pelos efeitos da retração do mercado, da redução das expectativas e do aumento de riscos observados na década de 1980.

This paper presents a study whose goal was to characterize the evolution of the agricultural modernization process in the Brazilian states. Factor analysis was applied to a group of variables related to the use of the main modern technologies. The results have led to the conclusion that the intensity of the modernization process was significantly different among the states. Since 1980, that process is becoming less intense, with the reduction in the values associated to the financing level and investments in agriculture, phenomenon related to the decrease in the offer of rural credit and the elevation

\footnotetext{
*Artigo recebido em set. 2001 e aprovado em set. 2002

** Professor associado no Laboratório de Engenharia Agrícola do Centro de Ciências e Tecnologias Agropecuárias da Universidade Estadual do Norte Fluminense (Uenf). E-Mail:pmsouza@uenf.br.

${ }^{* * *}$ Professor titular no Departamento de Economia Rural da Universidade Federal de Viçosa (UFV). E-mail: jelima@mail.ufv.br.
} 
of its cost. Possibly, this was also influenced by market retraction, lower expectations and increasing risks, events observed in the $1980 \mathrm{~s}$.

\section{Introdução}

O período compreendido entre meados dos anos 1960 até o final da década de 1970 caracteriza-se, segundo Buainain (1997), pela intervenção planejada, na qual a política agrícola se pautava por objetivos bastante claros, visando promover a expansão da oferta agropecuária, bem como a expansão e diversificação das exportações, e assegurar a normalidade do abastecimento doméstico. Esse modelo se direcionava para a modernização da base técnica, o fortalecimento da agroindústria e a expansão da fronteira agrícola, utilizando, para tanto, um conjunto de instrumentos que abrigava desde o crédito rural subsidiado, que foi central na política no período, até os programas de pesquisa agronômica e extensão rural, executados principalmente pela Empresa Brasileira de Pesquisa Agropecuária (Embrapa) e pela Empresa de Assistência Técnica e Extensão Rural (Embrater), respectivamente.

Esse período caracterizou a fase de modernização da agricultura brasileira, durante a qual um conjunto de instrumentos de política foi acionado para modernizar o setor. Tendo no crédito rural subsidiado um de seus principais indutores, esse processo resultou em grandes alterações na forma de produzir e nas relações desse setor com os demais segmentos da economia, promovendo a transformação da base técnica, com o incremento do uso de diversas inovações tecnológicas, como a mecanização e o emprego de diversos insumos industrializados.

Uma análise crítica desse processo de modernização da agricultura brasileira, levada a efeito por Silva et alii (1983), considera que o padrão tecnológico implantado direcionou-se basicamente para a expansão do complexo agroindustrial e não foi absorvido completamente pelos pequenos produtores, os quais, como resultado, se mantêm defasados em relação às unidades modernizadas. Nesse processo teve importância o crédito rural subsidiado, cujos benefícios foram maiores para a região Centro-Sul do que para a Norte-Nordeste, favorecendo essencialmente os produtos modernos e utilizadores de tecnologias mais avançadas, além de privilegiar os grandes produtores em detrimento dos pequenos.

Martine e Beskow (1987) salientam a importância de outras políticas que atuaram no período, como as dos preços mínimos, do seguro rural e de subsídios, acionadas a partir dos anos 1960, além dos programas especiais, criados no período de modernização da agricultura, como o Programa Nacional do Álcool (Proálcool) 
e vários outros. Esse conjunto de políticas e programas públicos atuou no sentido de incentivar a concentração fundiária, além de beneficiar atividades e regiões específicas em detrimento das demais e favorecer a grande produção. Somando-se a essa variedade de instrumentos, a política de extensão/assistência técnica, em especial a partir da criação do Sistema de Empresas Brasileiras de Assistência Técnica e Extensão Rural (Embrater/Emater/Aster), concentrou-se nas áreas geográficas e nos produtos de resposta mais rápida aos incentivos, marginalizando a parcela dos produtores ditos de baixa renda.

A falência desse modelo ocorreu nos anos 1980, devido à crise macroeconômica daquele momento e às mudanças estruturais e na conjuntura econômica, tudo isso evidenciando a necessidade de um novo paradigma de intervenção, como destacado por Buainain (1997). A favor dessa mudança de orientação, atuaram os altos custos incorridos pelo Tesouro Nacional na condução da política, o desequilíbrio entre a produção voltada para a exportação e a produção para o mercado interno, a alta instabilidade econômica no início dos anos 1980, a ineficiência dos instrumentos então vigentes para contornar riscos e incertezas e o alcance da modernização tecnológica, eliminando a necessidade dos tradicionais mecanismos utilizados para induzi-la.

O modelo seguinte, por sua vez, caracterizou-se por intervenções voltadas a solucionar problemas conjunturais, numa visão de curto prazo, com medidas nem sempre consistentes entre si e inconsistentes com um horizonte temporal mais longo. Nessa fase, foi estimulada a política de garantia de preços mínimos, concomitantemente com a contração do crédito subsidiado. Ao final da década de $1980 \mathrm{e}$ principalmente nos anos 1990, esse modelo também entrou em crise, em razão das inconsistências internas das políticas voltadas para o setor agrícola, bem como das inconsistências entre estas políticas setoriais e as ações no plano macroeconômico, mormente aquelas voltadas para a estabilização monetária (Buainain, 1997).

Nesse contexto, é esperado que o processo de modernização da agricultura caracterize-se por grande heterogeneidade entre as unidades da Federação e, por outro lado, que sua evolução tenha sofrido o impacto das mudanças nos mecanismos de intervenção governamental ocorridas no período, questões que são abordadas neste artigo.

\section{Objetivos}

O artigo visa fornecer evidências empíricas acerca do processo de modernização da agricultura, no Brasil e nas unidades da Federação, buscando verificar sua magnitude e descrever sua dinâmica ao longo do tempo. 


\section{Metodologia}

Admitindo como modernização da agricultura as mudanças ocorridas na base técnica de produção agropecuária, a aferição da magnitude e da evolução desse processo nos estados foi feita com o uso da análise fatorial, aplicada a um conjunto de variáveis relacionadas à utilização de tecnologias modernas. Esta medida possibilitou a descrição do processo de modernização nos estados, permitindo, ainda, por meio da análise de clusters, agrupá-los em classes distintas e mais ou menos homogêneas com respeito à importância desse processo.

\subsection{Análise fatorial}

Para estudar o processo de modernização da agricultura nas diversas unidades da Federação, fez-se uso da análise fatorial. Esta técnica tem sido adotada como instrumental de análise em vários estudos sobre a modernização da agricultura, como em Gomes (1990), Tarsitano (1992), Cardoso (1992), Hoffmann (1992), Cunha (1995), Meyer (1997) e Espírito-Santo (1998).

A análise fatorial refere-se a um conjunto de técnicas estatísticas cujo objetivo é representar um grupo de variáveis em termos de um menor número de variáveis hipotéticas (Kim e Mueller, 1978). De acordo com Schilderinck (1970), o método de análise fatorial consiste na tentativa de determinar as relações quantitativas entre as variáveis, de modo a associar, àquelas com padrão semelhante, o efeito de um fator causal subjacente e específico.

Para possibilitar a comparação, as $N$ observações das $n$ variáveis devem ser inicialmente normalizadas. A normalização consiste em expressar, em desvios padrões, os desvios das observações originais em relação à sua média. Cada variável normalizada $z_{i}(i=1,2, \ldots, n)$ deve ser relacionada separadamente às variáveis hipotéticas ou fatores $f_{j}(j=1,2, \ldots, m),(m<n, N)$. Essas relações são lineares e assumem, no modelo básico de análise fatorial, a seguinte expressão analítica (Harman, 1960):

$$
z_{i}=a_{i 1} f_{1}+a_{i 2} f_{2}+\ldots+a_{i m} f_{m}+d_{i} u_{i} \quad(i=1,2, \ldots, n)
$$

em que cada uma das $n$ variáveis é descrita, em termos lineares, como função dos $m$ fatores comuns $f_{j}$, aos quais se relacionam através das cargas fatoriais ou coeficientes de conexão $a_{i j}$, que indicam em que medida e direção as variáveis $z_{i}$ estão relacionadas com o fator $f_{j}$; e de um fator único $u_{i}$, que responde pela variância remanescente. 
Para saber se os fatores gerais causaram determinada relação entre as variâncias de $z_{i}$, é necessário que sua variância total $\left(\sigma_{i}^{2}\right)$ seja dividida em três componentes:

- a variância comum ou comunalidade, $h_{i}^{2}$, ou seja, que proporção da variância total de $z_{i}$ está associada com a variância das outras variáveis ou grupos de variáveis;

- a variância específica ou especificidade, $s_{i}^{2}$, isto é, a proporção da variância total que não mostra qualquer associação com a variância das outras variáveis;

- o erro ou distúrbio, $e_{i}^{2}$, que é a proporção da variância devida aos erros nas observações, ou a variáveis relevantes ao estudo, porém não consideradas no mesmo.

Os fatores únicos são sempre não-correlacionados com os fatores comuns, e, se estes últimos não são correlacionados entre si, a variância total de $z_{i}, \sigma_{i}^{2}$, pode ser expressa por:

$$
\sigma_{i}^{2}=a_{i 1}^{2}+a_{i 2}^{2}+\ldots+a_{i m}^{2}+d_{i}^{2}
$$

em que os componentes $a_{i j^{2}}$ são denominados percentagem de conexão e correspondem à proporção da variância total da variável normalizada $z_{i}$ que é explicada pelos respectivos fatores. Em (2), o termo

$$
h_{i}^{2}=a_{i 1}^{2}+a_{i 2}^{2}+\ldots+a_{i m}^{2}
$$

equivale à comunalidade da variável $z_{i}$, ao passo que o termo $d_{i^{2}}$ corresponde à unicidade, ou seja, a contribuição do fator único, indicando a extensão em que os fatores comuns falham na explicação da variância total da variável.

A unicidade pode ser decomposta em duas partes: uma devida à seleção das variáveis, denominada especificidade $\left(s_{i^{2}}\right)$, e outra atribuída à não-confiabilidade das medidas, denominada erro $\left(e_{i^{2}}\right)$ :

$$
d_{i}^{2}=s_{i}^{2}+e_{i}^{2}
$$

Com essa decomposição, o modelo linear (1) pode ser escrito na forma:

$$
z_{i}=a_{i 1} f_{1}+a_{i 2} f_{2}+\cdots+a_{i m} f_{m}+s_{i} S_{i}+e_{i} S_{i}
$$

em que $S_{i}$ e $E_{i}$ são os fatores específico e erro, respectivamente, e $s_{i}$ e $e_{i}$ são seus coeficientes. 
Para obtenção dos fatores, será empregado o método dos componentes principais, cujo princípio básico consiste em extrair fatores de modo a maximizar a contribuição dos mesmos para a comunalidade. Assim, um primeiro fator é escolhido para maximizar a soma dos quadrados das cargas fatoriais em relação a ele. Em seguida, obtém-se um segundo fator, para que também seja maximizada a soma de quadrados das cargas fatoriais em relação a ele, e assim por diante para os demais fatores.

\subsection{Análise de clusters}

Visando classificar as diversas unidades da Federação em termos dos fatores de modernização revelados pela análise fatorial, será empregada a técnica de análise de agrupamentos ou de clusters. A análise de clusters corresponde a um conjunto de métodos através dos quais se busca reunir os vários indivíduos em grupos, tipos ou classes, tomando como informações para classificação as medidas de um conjunto de variáveis, características ou atributos de cada indivíduo (Everitt, 1977). Conforme Duran e Odell (1974), o objetivo dessa analise é determinar, a partir das características dos indivíduos, subconjuntos tais que cada indivíduo pertença a um e somente um subconjunto, e que os indivíduos agrupados em um mesmo subconjunto sejam similares e aqueles pertencentes a grupos distintos sejam diferentes.

Para agrupar os indivíduos semelhantes, utilizam-se algoritmos que, como critério essencial, buscam maximizar as diferenças entre grupos relativas à variação dentro dos grupos. Os algoritmos mais utilizados podem ser classificados em hierárquicos e não-hierárquicos (Hair et alii, 1995). Os procedimentos não-hierárquicos designam os indivíduos para os grupos num processo em que o número de grupos deve ser previamente definido. Já os procedimentos hierárquicos consistem basicamente na formação de uma estrutura hierárquica e podem seguir a via aglomerativa ou divisiva.

Os métodos mais comumente empregados classificam-se no grupo das técnicas hierárquicas aglomerativas, em que a classificação dos indivíduos é feita mediante sucessivas fusões dos $n$ indivíduos em grupos. O procedimento básico consiste em computar uma matriz de distância ou similaridade entre os indivíduos, a partir da qual se inicia um processo de sucessivas fusões, com base na proximidade ou similaridade entre eles. Os resultados desse processo são passíveis de representação em um dendrograma, que nada mais é do que um diagrama bidimensional que exibe as fusões realizadas em cada nível, culminando no estágio em que todos os indivíduos estão num único grupo. 
Em razão do grande número de técnicas de agrupamento hierárquico disponíveis e das diferenças de resultados a que podem levar, Everitt (1977) sugere a adoção do procedimento de validação dos clusters obtidos, que consiste em aplicar, sobre os mesmos dados, várias técnicas de agrupamento e admitir como válidos apenas os clusters gerados pela maior parte dos métodos empregados. Esse foi o procedimento adotado neste artigo, no qual foram empregados os métodos de ligação simples (ou abordagem do vizinho mais próximo), ligação completa (ou abordagem do vizinho mais distante), centroid, ligação média e o método de Ward, os quais são, conforme Hair et alii (1995), os métodos aglomerativos mais comuns.

\subsection{Variáveis e fontes de dados}

Dado o caráter multidimensional do conceito de modernização agrícola, a magnitude desse processo nas unidades da Federação, bem como sua evolução no período estudado, requer a consideração de um conjunto de variáveis capazes de captar a utilização das tecnologias modernas a ele associadas.

Essas variáveis foram selecionadas tomando por base os diversos trabalhos abordando a modernização agrícola e tentam captar a importância da utilização de produtos industriais na agricultura, bem como o crescimento da produtividade dos fatores de produção.

Nesse sentido, indicadores relativos às despesas com insumos diversos e aos investimentos em instalações e outros bens visam apreender o crescente gasto com insumos e o crescimento da interdependência setorial, bem como a capitalização da agricultura.

Foram considerados também indicadores relativos ao valor dos financiamentos, em grande parte responsáveis pelas transformações ocorridas na agricultura. O crédito rural subsidiado, principal instrumento da política de modernização, passou por mudanças ao longo do período de estudo, com elevação das taxas de juros e redução de oferta a partir da década de 1980. Uma vez que essas mudanças afetam, em última instância, o volume de financiamentos, com possíveis reflexos na intensidade de modernização da agricultura, acredita-se que essa variável seja suficiente para captar as mudanças ocorridas na política de crédito rural nos anos 1980.

Uma vez que não é o volume, mas a intensidade do uso das tecnologias modernas o aspecto de interesse, a maioria das variáveis é expressa em relação à mão-de-obra ocupada, em equivalentes-homem-ano $(E H)$, e em relação à área explorada $(A E)$. De acordo com Hoffmann (1992), enquanto a área trabalhada $(A T)$ equivale à soma das áreas ocupadas com lavouras permanentes e temporárias 
e com pastagens e matas plantadas, o conceito de área explorada $(A E)$ envolve, em adição às atividades admitidas na área trabalhada, as áreas cobertas por pastagens e matas naturais.

Para descrever o processo de modernização, as seguintes variáveis foram empregadas:

- $X 1$ = proporção da área com pastagem que é plantada;

- $X 2$ = percentagem dos estabelecimentos que utilizam força mecânica;

- $X 3=$ número de tratores $/ A E$;

- $X 4$ = número de tratores $/ E H$;

- $X 5$ = número de arados de tração mecânica/AE;

- $X 6$ = número de arados de tração mecânica/EH;

- $X 7=$ número de máquinas para colheita/AE;

- $X 8$ = número de máquinas para colheita/EH;

- $X 9=$ valor total dos investimentos $/ A E$;

- $X 10=$ valor dos investimentos em instalações e outras benfeitorias $/ A E$;

- $X 11=$ valor dos investimentos em veículos e outros meios de transporte/AE;

- $X 12$ = valor dos investimentos em máquinas e instrumentos agrários/AE;

- $X 13=$ valor total dos investimentos $/ E H$;

- $X 14=$ valor dos investimentos em instalações e outras benfeitorias $/ E H$;

- $X 15=$ valor dos investimentos em veículos e outros meios de transporte/EH;

- $X 16$ = valor dos investimentos em máquinas e instrumentos agrários/EH;

- $X 17=$ valor total dos financiamentos obtidos $/ A E$;

- $X 18=$ valor total dos financiamentos obtidos $/ E H$;

- $X 19$ = valor dos financiamentos em relação ao valor da produção;

- $X 20=$ valor da produção/AE; 
- $X 21$ = valor da produção/EH;

- $X 22=$ despesa total $/ A E$;

- $X 23=$ despesas com adubos e corretivos $/ A E$;

- $X 24=$ despesas com sementes e mudas $/ A E$;

- $X 25=$ despesas com defensivos agrícolas $/ A E$;

- $X 26=$ valor total dos combustíveis consumidos/AE;

- $X 27=$ despesa total $/ E H$;

- $X 28=$ despesas com adubos e corretivos $/ E H$

- $X 29=$ despesas com sementes e mudas $/ E H$;

- $X 30=$ despesas com defensivos agrícolas $/ E H$;

- $X 31=$ valor total dos combustíveis consumidos $/ E H$

- $X 32=$ percentagem dos estabelecimentos que utilizam energia elétrica.

Essas variáveis foram calculadas a partir de informações disponíveis nos censos agropecuários da Fibge. Todos os valores monetários foram uniformizados e convertidos em reais de 1994, utilizando-se o índice geral de preços disponibilidade interna, IGP-DI, da Fundação Getulio Vargas (base $1994=100$ ).

\section{Resultados e Discussão}

\subsection{Fatores de modernização}

Uma vez que o interesse da pesquisa recai sobre a dinâmica do processo de modernização, a análise fatorial foi conduzida agregando-se as observações feitas para os 32 indicadores, nos cinco anos considerados. Isso porque, se a análise fatorial fosse realizada para cada ano individualmente, os fatores obtidos num ano não seriam idênticos aos do ano seguinte, o que inviabilizaria qualquer tentativa de verificar o comportamento da modernização agrícola ao longo do período estudado. Desse modo, a análise incidiu sobre a matriz $M$, de dimensão $130 \times 32$, em que: 


$$
M=\left[\begin{array}{l}
M 1 \\
M 2 \\
M 3 \\
M 4 \\
M 5
\end{array}\right]
$$

sendo $M 1, M 2, M 3, M 4$ e $M 5$ as matrizes de ordem $26 \times 32$, referentes aos anos de 1970, 1975, 1980, 1985 e 1995, respectivamente, e que são formadas pelos valores dos 32 indicadores de modernização, observados em cada uma das 26 unidades de Federação. ${ }^{1}$ As observações foram ponderadas pelo valor da produção agropecuária da unidade da Federação, visando captar as diferenças de contribuição para a produção total do país. Para a obtenção dos pesos, dividiu-se o valor da produção de cada unidade da Federação, em cada ano, pela média aritmética do valor da produção do conjunto de unidades da Federação no respectivo ano, como sugerido por (Hoffmann, 1992).

Uma vez constituída, a matriz $M$ foi submetida à análise fatorial, através do programa estatístico Statistical Package Software (SPSS 8.0).

Para determinar se a análise fatorial é apropriada para o estudo dos dados empregados, Hair Jr. sugere o uso de medidas que consideram toda a matriz de correlação entre as variáveis (Hair et alii, 1995). Este é o caso do teste de esfericidade de Bartlett, um teste estatístico para determinar a presença de correlações entre as variáveis, que fornece a probabilidade estatística de que a matriz de correlações tenha correlações significativas entre pelo menos algumas das variáveis. Este teste foi realizado, e o valor obtido $(15.493,11)$ mostra-se significativo a $1 \%$ de probabilidade, ou seja, permite rejeitar a hipótese nula de que a matriz de correlação é uma matriz identidade, isto é, de que as variáveis não são correlacionadas.

Outra forma de quantificar o grau de intercorrelações entre as variáveis e a adequação da análise fatorial ao conjunto de dados, sugerida pelo mesmo autor, é a medida de adequação da amostra. Esta medida pode assumir valores entre 0 e 1, atingindo a unidade quando cada variável é perfeitamente predita pelas demais.

\footnotetext{
${ }^{1}$ Dada a impossibilidade de reconstituir as informações para o estado de Tocantins, criado em 1988 a partir do desmembramento do estado de Goiás, optou-se por considerar apenas o antigo estado de Goiás, que, por conseguinte, engloba a área hoje ocupada por Tocantins - daí a presença de apenas 26 unidades da Federação. Já para Mato Grosso e Mato Grosso do Sul, separados em 1979, essa reconstituição foi possível. Como as informações desmembradas já se encontravam disponíveis no censo agropecuário de 1975, optou-se por reconstituir indicadores de modernização para cada estado no ano de 1970, distribuindo os valores deste ano entre os mesmos, mantendo-se as proporções com que figuravam no ano de 1975.
} 
No estudo apresentado neste artigo, o teste de Kaiser-Meyer-Olkim foi empregado como medida de adequação da amostra, e o valor obtido foi 0,87 . Conforme a classificação fornecida por Hair et alii (1995), valores acima de 0,5 indicam que a amostra é adequada a esse tipo de análise, com os valores acima de 0,80 permitindo classificar a adequação como acima da média ou meritória, sendo esta a situação em que se encontram os dados empregados no estudo.

Desse modo, ambos os testes realizados permitiram concluir que a amostra utilizada é adequada ao procedimento de análise, ou seja, ao emprego da análise fatorial.

A análise através do método dos componentes principais gerou três fatores com raízes características maiores que 1 , como pode ser observado na tabela 1 .

Tabela 1

Fatores obtidos pelo método dos componentes principais

\begin{tabular}{cccc}
\hline Fator & $\begin{array}{c}\text { Raiz } \\
\text { característica }\end{array}$ & $\begin{array}{c}\text { Variância explicada } \\
\text { pelo fator }(\%)\end{array}$ & $\begin{array}{c}\text { Variância } \\
\text { acumulada }(\%)\end{array}$ \\
\hline 1 & 26,64 & 83,25 & 83,25 \\
2 & 1,48 & 4,63 & 87,89 \\
3 & 1,25 & 3,91 & 91,80 \\
\hline Fonte: Dados da pesquisa. & &
\end{tabular}

A contribuição dos fatores 1, 2 e 3 para a explicação da variância total dos indicadores utilizados é de $83,25 \%, 4,63 \%$ e $3,91 \%$, respectivamente, de modo que a contribuição acumulada dos mesmos equivale a 91,80\%. Uma vez que inexiste um critério para definir qual o número de fatores principais que devem ser extraídos, optou-se pela caracterização do processo de modernização agrícola nas unidades da Federação através dos dois primeiros fatores, que, conjuntamente, explicam 87,89\% da variância total das variáveis analisadas. Muito embora a opção por maior número de fatores possibilite explicar uma proporção mais elevada da variância dos indicadores, a opção por dois fatores permite que a posição de cada unidade de Federação, em dado ano, possa ser representada em um sistema de coordenadas cartesianas com dois eixos.

Para facilitar a interpretação dos fatores, estes foram submetidos a uma rotação ortogonal pelo método Varimax, sugerido por Kim e Mueller (1978). Com esse procedimento, a contribuição de cada fator para a variância total é alterada, sem, contudo, modificar a contribuição conjunta dos mesmos. Como vantagem, os fatores obtidos após a rotação encontram-se mais estreitamente relacionados a determinados grupos de variáveis, possibilitando uma interpretação mais lógica dos mesmos. 
A tabela 2 exibe as cargas fatoriais, as comunalidades e o percentual da variância total dos indicadores que é explicado por cada fator, após a rotação. A contribuição dos fatores $F 1$ e $F 2$ para a explicação da variância total dos indicadores utilizados é de $47,60 \%$ e 40,28\%, respectivamente. Para melhor interpretação, as cargas fatoriais com valor superior a 0,700 estão em negrito, buscando evidenciar os indicadores mais fortemente associados a determinado fator.

Tabela 2

Cargas fatoriais após a rotação ortogonal e comunalidades obtidas na análise fatorial dos indicadores de modernização da agricultura nas unidades de Federação (1970-95)

\begin{tabular}{|c|c|c|c|}
\hline \multirow{2}{*}{ Variável } & \multicolumn{2}{|c|}{ Carga Fatorial } & \multirow{2}{*}{ Comunalidades } \\
\hline & $F 1$ & $F 2$ & \\
\hline $\mathrm{X} 1$ & 0,490 & 0,738 & 0,785 \\
\hline $\mathrm{X} 2$ & 0,522 & 0,808 & 0,926 \\
\hline X3 & 0,465 & 0,848 & 0,936 \\
\hline $\mathrm{X} 4$ & 0,357 & 0,901 & 0,939 \\
\hline X5 & 0,697 & 0,445 & 0,684 \\
\hline $\mathrm{X} 6$ & 0,640 & 0,519 & 0,680 \\
\hline $\mathrm{X} 7$ & 0,789 & 0,268 & 0,695 \\
\hline $\mathrm{X} 8$ & 0,756 & 0,304 & 0,664 \\
\hline X9 & 0,798 & 0,541 & 0,929 \\
\hline $\mathrm{X} 10$ & 0,766 & 0,582 & 0,925 \\
\hline $\mathrm{X} 11$ & 0,800 & 0,537 & 0,929 \\
\hline $\mathrm{X} 12$ & 0,810 & 0,443 & 0,852 \\
\hline $\mathrm{X} 13$ & 0,685 & 0,549 & 0,771 \\
\hline $\mathrm{X} 14$ & 0,668 & 0,619 & 0,829 \\
\hline $\mathrm{X} 15$ & 0,751 & 0,597 & 0,921 \\
\hline $\mathrm{X} 16$ & 0,789 & 0,509 & 0,883 \\
\hline $\mathrm{X} 17$ & 0,886 & 0,398 & 0,943 \\
\hline $\mathrm{X} 18$ & 0,849 & 0,452 & 0,924 \\
\hline X19 & 0,836 & 0,296 & 0,786 \\
\hline $\mathrm{X} 20$ & 0,702 & 0,664 & 0,933 \\
\hline $\mathrm{X} 21$ & 0,635 & 0,757 & 0,977 \\
\hline $\mathrm{X} 22$ & 0,663 & 0,716 & 0,952 \\
\hline $\mathrm{X} 23$ & 0,758 & 0,614 & 0,952 \\
\hline $\mathrm{X} 24$ & 0,753 & 0,520 & 0,838 \\
\hline $\mathrm{X} 25$ & 0,592 & 0,755 & 0,920 \\
\hline $\mathrm{X} 26$ & 0,750 & 0,627 & 0,957 \\
\hline $\mathrm{X} 27$ & 0,582 & 0,791 & 0,965 \\
\hline $\mathrm{X} 28$ & 0,693 & 0,675 & 0,936 \\
\hline X29 & 0,705 & 0,628 & 0,892 \\
\hline X30 & 0,503 & 0,848 & 0,971 \\
\hline X31 & 0,675 & 0,690 & 0,931 \\
\hline X32 & 0,207 & 0,925 & 0,899 \\
\hline da variância & 47,60 & 40,28 & \\
\hline
\end{tabular}

Na tabela 2, pode-se constatar que o fator 1 encontra-se mais fortemente corre- 
lacionado com as variáveis número de máquinas para colheita/ $\underline{A E}(X 7)$, número de máquinas para colheita/EH $(X 8)$, valor total dos investimentos/AE $(X 9)$, valor dos investimentos em instalações e outras benfeitorias/ $A E(X 10)$, valor dos investimentos em veículos e outros meios de transporte/ $A E$ (X11), valor dos investimentos em máquinas e instrumentos agrários/ $A E$ (X12), valor dos investimentos em veículos e outros meios de transporte/EH (X15), valor dos investimentos em máquinas e instrumentos agrários/EH (X16), valor total dos financiamentos obtidos/AE (X17), valor total dos financiamentos obtidos/EH (X18), valor dos financiamentos em relação ao valor da produção $(X 19)$, valor da produção/ $A E$ $(X 20)$, despesas com adubos e corretivos/AE (X23), despesas com sementes e mudas/ $A E(X 24)$, valor total dos combustíveis consumidos/AE (X26) e despesas com sementes e mudas/EH (X29). Considerando-se cargas fatoriais superiores a 6 , pode-se constatar que esse fator apresenta também alta correlação com as variáveis número de arados de tração mecânica/ $A E(X 5)$, número de arados de tração mecânica/ $E H(X 6)$, valor total dos investimentos/ $E H(X 13)$, valor dos investimentos em instalações e outras benfeitorias/ $E H(X 14)$ e despesas com adubos e corretivos/EH (X28).

Já o segundo fator relaciona-se mais estreitamente com o conjunto de variáveis proporção da área com pastagem que é plantada $(X 1)$, percentagem dos estabelecimentos que utilizam força mecânica $(X 2)$, número de tratores/ $A E(X 3)$, número de tratores/EH (X4), valor da produção/EH (X21), despesa total/AE (X22), despesas com defensivos agrícolas/AE (X25), despesa total/EH (X27), despesas com defensivos agrícolas/EH $(X 30)$, percentagem dos estabelecimentos que utilizam energia elétrica $(X 32)$ e também valor total dos combustíveis consumidos/EH (X31), com carga fatorial próxima a 0,70 .

É importante destacar que, mesmo após a rotação, algumas variáveis, ainda que mais fortemente correlacionadas com um dos fatores, apresentam também elevada correlação com o outro fator.

Assim, as variáveis valor dos investimentos em instalações e outras benfeitorias/EH (X14), valor da produção/AE (X20), despesas com adubos e corretivos/AE (X23), valor total dos combustíveis consumidos/AE (X26), despesas com adubos e corretivos/EH (X28) e despesas com sementes e mudas/EH (X29), ainda que mais fortemente correlacionadas ao primeiro fator, apresentam também elevada correlação com o fator 2 .

De modo semelhante, as variáveis valor total dos combustíveis consumidos/ $E H$ (X31), valor da produção/EH (X21) e despesa total/AE (X22), que se correlacionam mais fortemente com o fator 2 , apresentam também carga fatorial elevada com o fator 1 . 
Em razão disso, torna-se importante, no intuito de estabelecer uma interpretação desses fatores, minimizar o significado associado às variáveis não estritamente relacionadas a um único fator especificamente. Por exemplo, as variáveis que captam as produtividades da terra $(X 20)$ e da mão-de-obra $(X 21)$, ainda que mais fortemente correlacionadas aos fatores 1 e 2, respectivamente, apresentam cargas fatoriais elevadas com ambos os fatores. Nesse sentido, a vinculação de qualquer dessas variáveis a um fator específico pode conduzir a interpretações errôneas, principalmente nas situações em que os fatores não variem na mesma direção, quando então seria impreciso dizer o sentido predominante daquelas variáveis.

Desse modo, pode-se constatar que as variáveis mais forte e estreitamente correlacionadas ao fator 1 resumem-se a: número de máquinas para colheita/ $A E$ $(X 7)$, número de máquinas para colheita/EH (X8), valor total dos investimentos/AE (X9), valor dos investimentos em instalações e outras benfeitorias/AE $(X 10)$, valor dos investimentos em veículos e outros meios de transporte/ $A E$ (X11), valor dos investimentos em máquinas e instrumentos agrários/ $A E(X 12)$, valor dos investimentos em veículos e outros meios de transporte/ $E H$ (X15), valor dos investimentos em máquinas e instrumentos agrários/ $E H(X 16)$, valor total dos financiamentos obtidos/AE (X17), valor total dos financiamentos obtidos/EH (X18), valor dos financiamentos em relação ao valor da produção $(X 19)$, despesas com sementes e mudas/AE (X24) e, com cargas fatoriais superiores a 0,600 , número de arados de tração mecânica/ $A E(X 5)$, número de arados de tração mecânica/EH (X6) e valor total dos investimentos/EH (X13).

Portanto, o fator 1 está mais estreitamente relacionado com todas as variáveis que captam a intensidade de uso de financiamentos e a magnitude dos investimentos totais, bem como com algumas variáveis relativas ao emprego de colheitadeiras, arados de tração mecânica e despesas com sementes e mudas. Pode-se dizer que esse fator sintetiza o processo, dependente e induzido por um crescente volume de financiamentos, de elevação dos investimentos totais na agricultura e, particularmente, em instalações, benfeitorias, veículos, máquinas e equipamentos. Por simplificação, esse fator será denominado nível de financiamentos e de investimentos na agricultura.

O fator 2, por sua vez, encontra-se, de fato, mais estreitamente relacionado aos indicadores proporção da área com pastagem que é plantada $(X 1)$, percentagem dos estabelecimentos que utilizam força mecânica $(X 2)$, percentagem dos estabelecimentos que utilizam energia elétrica $(X 32)$, número de tratores/ $A E(X 3)$, número de tratores/ $E H(X 4)$, despesa total/EH $(X 27)$, despesas com defensivos agrícolas/AE (X25) e despesas com defensivos agrícolas/EH (X30).

Esse fator está mais fortemente correlacionado com os indicadores direta ou 
indiretamente ligados ao uso de tecnologias modernas e dependente de fontes nãotradicionais de energia, como os indicadores relativos ao emprego de tratores $(X 3$ e $X 4$ ), ao uso da força mecânica $(X 2)$ e à utilização de energia elétrica (X32). Apresenta-se também correlacionado à despesa total $(X 27)$ e aos gastos com defensivos agrícolas (X25 e X30), estando ainda fortemente correlacionado com a proporção da área com pastagem que é plantada $(X 1)$. Por simplificação, esse fator será denominado uso de tratores e de fontes não-tradicionais de energia e despesas com a produção.

\subsection{A dinâmica da modernização agrícola no período 1970-95}

Obtidas as cargas fatoriais, o passo seguinte consistiu na determinação dos escores fatoriais, ou seja, do valor dos fatores obtidos para cada região e ano. Para tanto, obteve-se, num primeiro momento, a matriz de coeficientes fatoriais, resultado da multiplicação da matriz de cargas fatoriais pela inversa da matriz de correlação. Através da multiplicação da matriz de coeficientes fatoriais pela matriz de dados originais padronizados, foram calculados os escores fatoriais. Mediante uma média dos escores das unidades da Federação, ponderada pelas respectivas participações no valor da produção, foram obtidos os valores dos fatores para o Brasil. Os resultados desse procedimento encontram-se no anexo deste artigo.

De posse dos escores fatoriais, procurou-se obter alguma classificação das unidades de Federação, reunindo em grupos as unidades semelhantes em termos da magnitude da modernização e da forma como esta se processou. Para tanto, optou-se pela análise de clusters, mediante a qual as unidades de Federação são agrupadas, tomando-se como informações os valores dos escores fatoriais ao longo dos anos estudados.

Para atribuir, no processo de agrupamento, maior expressão aos fatores capazes de melhor sintetizar o processo de modernização, optou-se por uma ponderação dos escores fatoriais. Muito embora esse procedimento seja controverso e sujeito a críticas, tendo como principal argumento contrário o de que os pesos possam basear-se apenas em julgamentos intuitivos, como levantado em Everitt (1977), este não é o caso presente, uma vez que a contribuição de cada fator de modernização para a variância total dos dados é, por si mesma, evidência da importância relativa de cada um deles.

Assim, os escores fatoriais foram ponderados utilizando-se pesos que refletissem a contribuição relativa de cada fator para a explicação da variância total, de modo a assegurar que o agrupamento levasse essas diferenças em consideração. A partir dos dados da última linha da tabela 2 , pode-se constatar que a contri- 
buição total dos fatores para a variância é distribuída na proporção de 0,54 e 0,46, respectivamente para os fatores 1 e 2. Assim, multiplicando-se a participação de cada fator na variância por 2 (número de fatores), obtêm-se os valores 1,08 e 0,92, para os fatores 1 e 2, nessa ordem. Esses valores foram empregados como pesos na ponderação.

Os resultados da análise de clusters, combinados com a observação do comportamento dos fatores de modernização $F 1$ e $F 2$, definido num sistema de dois eixos ortogonais, permitiram distribuir as unidades da Federação, selecionados de acordo com o padrão da modernização agrícola que seguiram, em quatro grupos distintos:

- grupo I - Acre, Amapá, Amazonas, Pará, Piauí e Rondônia;

- grupo II - Bahia, Ceará, Maranhão, Paraíba, Pernambuco, Rio Grande do Norte, Roraima e Sergipe;

- grupo III - Alagoas, Espírito Santo, Goiás, Mato Grosso, Minas Gerais e Rio de Janeiro;

- grupo IV - Distrito Federal, Mato Grosso do Sul, Paraná, Rio Grande do Sul, Santa Catarina e São Paulo.

Uma vez distinguidos esses grupos, o passo seguinte consistiu em verificar, através do comportamento dos fatores 1 (nível de financiamentos e de investimentos na agricultura) e 2 (uso de tratores e de fontes não- tradicionais de energia e despesas com a produção), a dinâmica da modernização agrícola entre os estados. Esse comportamento pode ser observado nas figuras 1 a 4 , nas quais o avanço da modernização, em cada estado, é descrito por cinco pontos, representando os anos de 1970, 1975, 1980, 1985 e 1995, os quais são unidos por setas orientadas com origem no ano de 1970.

A figura 1 exibe o comportamento da modernização agrícola no período 197095 nas unidades da federação pertencentes ao grupo I. Embora exista uma certa dificuldade em estabelecer uma medida que caracterize a magnitude da modernização que cada unidade da Federação atingiu, é possível, com base nos valores dos fatores, nos clusters obtidos e na representação gráfica dos escores, fazer alguma distinção entre os grupos formados quanto à intensidade do processo de modernização. Nesse sentido, pode-se dizer que o grupo I, composto principalmente por estados da região Norte, exceto Piauí, é aquele em que o processo de modernização foi menos intenso. Na figura 1, pode-se notar que esse processo é descrito 
por pontos que, na maior parte do tempo, estão situados no terceiro quadrante, que caracteriza valores negativos para ambos os fatores.

No que diz respeito à evolução do processo entre os anos estudados, podese constatar, na figura l que, para Acre, Amapá e Piauí, o período 1970-75 caracterizou-se por crescimento do fator 1 , concomitantemente ao declínio do segundo fator, observando-se situação oposta para Amazonas, Pará e Rondônia. No qüinqüênio seguinte, Amapá, Amazonas, Piauí e Rondônia experimentam crescimento no primeiro fator, associado a declínio do fator 2 , o contrário ocorrendo no Acre e Pará.

No período 1980-85, observa-se, para quase todas as unidades da Federação desse grupo, um movimento de declínio do fator 1 e de aumento do fator 2 , o que somente não se verifica no Pará, onde as mudanças se deram no sentido inverso. No restante do período, a tendência de decréscimo do fator 1 e de crescimento do fator 2 perdura, sendo observada para o conjunto dos estados, sem exceções.

Figura 1

Modernização da agricultura nas unidades da Federação do grupo I (1970-95)

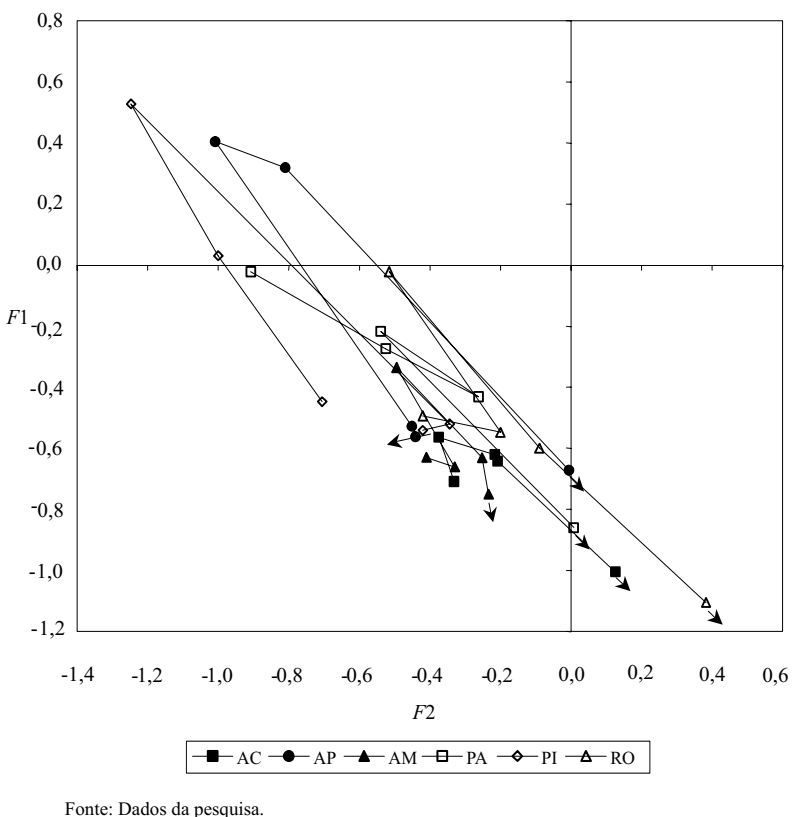

A figura 2 apresenta a evolução dos fatores de modernização para as unidades da Federação do grupo II, em geral pertencentes à região Nordeste. Pode-se cons- 
tatar que, em relaçao aos estados do grupo I, é maior a intensidade do processo de modernização nos estados desse grupo. Pode-se observar que, para a maior parte dos estados pertencentes a esse grupo, a década de 1970 foi marcada por um processo de crescimento do fator 1 e de declínio do fator 2. As exceções ficam por conta dos estados da Bahia e de Roraima, onde, na segunda metade da década, ocorreu também crescimento do segundo fator, e de Sergipe, onde, nesse mesmo período, observou-se o declino do primeiro fator e crescimento do segundo.

Figura 2

Modernização da agricultura nas unidades da Federação do grupo II (1970-95)

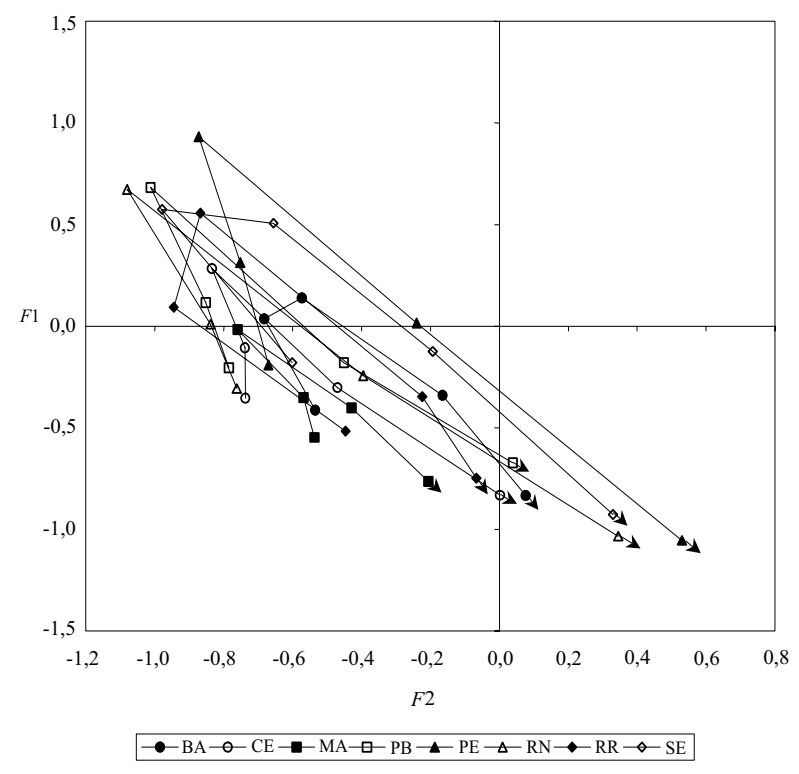

Fonte: Dados da pesquisa.

A partir de 1980 verifica-se uma reversão do comportamento até então observado. Contrariamente ao que fora observado no decênio anterior, a partir desse momento ocorre decréscimo no valor do primeiro fator, acompanhado de um retorno ao crescimento do fator 2. Esse movimento, que perdurou ao longo de todo o período restante da série analisada, ocorreu em todas as unidades da Federação pertencentes a esse grupo.

O comportamento da modernização nas unidades da Federação do terceiro grupo pode ser observado na figura 3. Pode-se concluir que os elementos desse grupo experimentaram um processo de modernização bem mais intenso do que 
aquele ocorrido nos grupos anteriores, sendo que, em geral, as coordenadas que o descrevem encontram-se, na maior parte do período, com exceção do ano de 1970, situadas no primeiro, segundo e quarto quadrantes, onde ambos ou pelo menos um dos fatores é positivo.

Figura 3

Modernização da agricultura nas unidades da Federação do grupo III (1970-95)

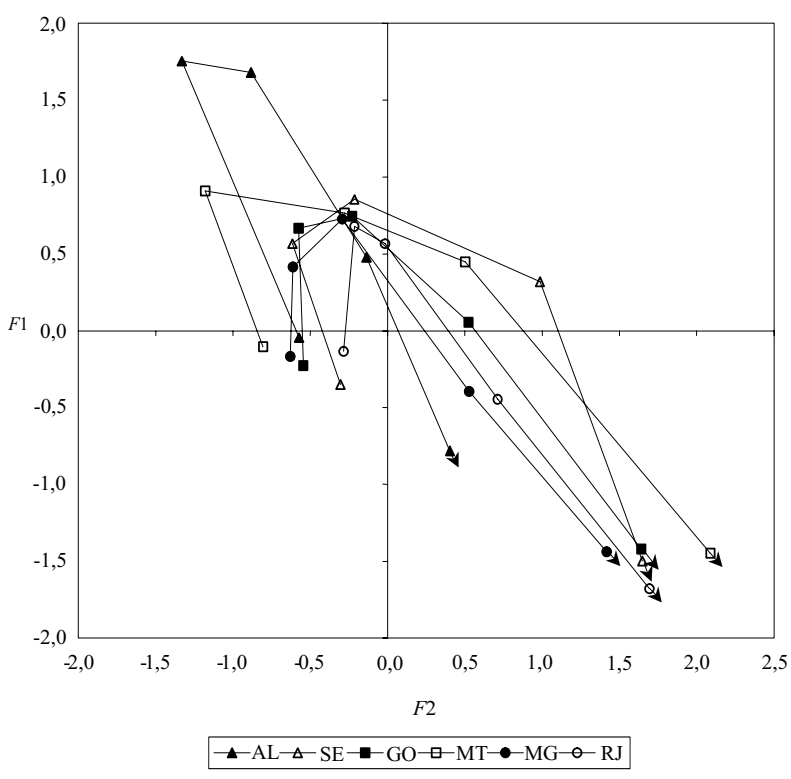

Fonte: Dados da pesquisa

Este grupo é constituído por estados das regiões Sudeste e Centro-Oeste, com exceção de Alagoas, onde a maior intensidade da modernização pode estar relacionada ao avanço do cultivo da cana-de-açúcar, como revelam Campos et alii (1988).

Pode-se observar que, entre 1970 e 1975, todos os estados desse grupo experimentaram um crescimento do fator 1 , em geral acompanhado de redução do segundo fator, não observada apenas em Minas Gerais e no Rio de Janeiro. Na segunda metade da década, mantém-se a tendência de incremento do fator 1 nos estados do Espírito Santo, de Goiás e de Minas Gerais, mas não mais em Alagoas, Mato Grosso e Rio de Janeiro. Esse período assinala ainda um crescimento do fator 2, que é observado em todas os estados desse grupo.

Para todas as unidades da Federação desse grupo, o período entre 1980 e 1995 
é caracterizado pela manutenção do crescimento do fator 2 , que já se iniciara na segunda metade da década de 1970, concomitantemente a uma redução persistente no valor do primeiro fator.

A figura 4 exibe o comportamento dos fatores para as unidades da Federação do grupo IV, que podem ser admitidas como aquelas em que a modernização agrícola foi mais intensa. Nessa figura, estão também plotados os valores dos fatores de modernização do país, obtidos mediante ponderação dos escores encontrados para as unidades da Federação. Nesse grupo, o processo de modernização seguiu, quase sem exceções, um mesmo padrão ao longo do tempo. Esse padrão se caracteriza, de início, por um crescimento do primeiro fator, acompanhado de pequena elevação do fator 2. Entre 1975 e 1980, mantém-se em elevação o fator 1, havendo, entretanto, pequeno declínio do fator 2, exceto para Mato Grosso do Sul e Santa Catarina, estados onde ambos os fatores experimentam crescimento no período. A partir de 1980, inicia-se um processo de declínio do fator 1, acompanhado de crescimento do segundo fator, comportamento que se mantém durante todo o restante do período analisado, sendo observado no Brasil e nas demais unidades da Federação desse grupo.

\section{Figura 4}

Modernização da agricultura nas unidades da Federação do grupo IV (1970-95)

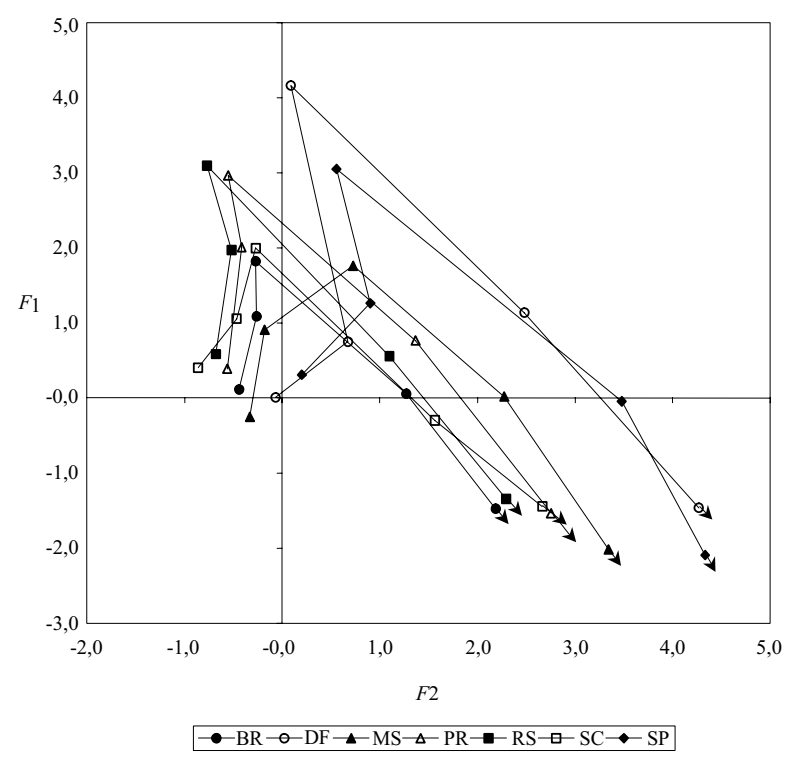

Fonte: Dados da pesquisa. 
Portanto, da análise efetuada para cada um dos grupos, pode-se concluir que, para o conjunto das unidades da Federação e para a agricultura do país como um todo, a modernização agrícola, conquanto se tenha processado em diferentes intensidades, seguiu um padrão comum facilmente identificável.

Assim, o que se constata é que a maioria das unidades da Federação passou, até 1980 , por um processo de modernização agrícola no qual inicialmente houve incremento do fator 1, que define o maior nível de financiamentos e de investimentos no setor, elevando a importância dos ativos fixos nele aplicados, como colheitadeiras, máquinas e veículos de transporte, instalações e outras benfeitorias. Entretanto, para a maior parte das unidades da Federação, é só a partir de 1980, principalmente, que a modernização se faz sentir também no avanço do fator 2, que caracteriza o uso de força mecânica, de energia elétrica e de tratores, bem como a elevação nas despesas envolvidas na condução do processo produtivo, sem as quais o mesmo não pode prosseguir.

Assim, se em todo o período compreendido entre 1970 e 1980 se verifica, para todas as unidades da Federação, um aumento no nível de financiamento e investimentos na agricultura, é somente a partir de 1980 que esta parece exibir uma nova face, perdendo sua auto-suficiência e caminhando no sentido de maior dependência do aporte de insumos externos à produção, e elevando o nível de despesas necessárias à condução da mesma.

Por outro lado, o período posterior a 1980 é caracterizado por um movimento de declínio do fator 1 e, portanto, do nível de financiamento e investimentos na agricultura, observado em todas as unidades da Federação, persistindo até o final do período estudado. Como há um conjunto de outras variáveis que, embora fortemente correlacionadas a esse fator, estão também, em grande medida, correlacionadas ao fator 2, que apresenta grande crescimento após 1980, certamente a redução do primeiro fator não pode ser entendida como queda de todos os indicadores a ele fortemente correlacionados. A interpretação possível é a de que houve, certamente, redução dos indicadores relativos ao uso de financiamentos e ao volume de investimentos na agricultura, não se podendo afirmar ter havido regressão no processo de modernização, mas apenas redução na intensidade com que vinha ocorrendo até então.

Esses resultados estão de acordo com os obtidos por Aguirre e Bacha (1989), segundo os quais a quantidade de insumos e máquinas não teve, na década de 1980, o mesmo ritmo de crescimento da década anterior. Também Gasques e Conceição (1998), analisando a evolução da produtividade total dos fatores na agricultura em seis estados (Rio de Janeiro, Espírito Santo, Santa Catarina, Goiás, Tocantins e Rio Grande do Norte), mostram que, embora não tenha ocorrido redução na 
produtividade total entre 1985 e 1995, seu ritmo de crescimento foi relativamente menor que o obtido no período de 1970 a 1985, que marcou a fase de consolidação do processo de modernização agrícola.

Embora obtendo fatores com diferentes significados, resultados obtidos por Meyer (1997) em relação à modernização da agricultura mineira indicam ter ocorrido, na primeira metade da década de 1980, uma desaceleração do processo de intensificação do uso de tecnologias modernas por área explorada, e mesmo redução do emprego de tecnologias modernas e de capital em relação ao pessoal ocupado.

Também Figueiredo e Hoffmann (1998), ao analisarem a dinâmica da modernização agrícola em 299 microrregiões homogêneas do Brasil, concluíram que, entre 1980 e 1985, último ano estudado, houve desaceleração da tecnificação da agricultura, decorrente do efeito das políticas recessivas do início dos anos 1980, que culminaram em restrições ao financiamento da agropecuária.

De acordo com Manoel e Barros (1987), a redução no ímpeto da modernização agrícola se deve à erosão das expectativas e ao aumento de riscos na atividade agrícola, que tiveram por efeito deprimir a demanda de investimentos neste setor. Por outro lado, a ocorrência de redução expressiva do dinamismo geral das atividades agropecuárias e a drástica queda no ritmo de incorporação tecnológica verificada entre 1980 e 1985 é admitida por Martine (1989) como o resultado da exaustão do padrão de financiamento vigente até o final de década de 1970 e da retração do mercado, decorrentes da crise econômica desse período.

A partir de 1982 observou-se redução no volume de financiamento das atividades agrícolas, que atingiu o nível mais baixo nos anos de 1984 e 1985, marcados pela redução do nível de subsídio embutido no crédito. Esses subsídios, medidos pela diferença entre a taxa de juros cobrada e a taxa de inflação, cresceram ao longo da década de 1970, atingindo, em 1980, o valor de 38,5\%. Com a aplicação de mecanismos de indexação pelo governo, esses subsídios, de 36,3\% em 1983, descem a 1,6\% em 1984, com as taxas de juros chegando, em 1985, a um valor real de $3,6 \%$ (Coelho, 1998).

Com o Plano Cruzado, o ano de 1986 marca um retorno ao crescimento dos financiamentos, captados a taxas reais de $7,3 \%$, movimento em parte explicado pelo impacto psicológico das taxas nominais de juros reduzidas. Compensado pelo comportamento favorável dos preços agrícolas e pela atuação de outros instrumentos, como a política de garantia de preços mínimos e o Programa de Garantia da Atividade Agropecuária (Proagro), o volume de crédito tomado não decresceu drasticamente após 1986, em face da oferta ainda abundante de recursos. Somente a partir de 1990 esse volume decresce mais intensamente, com o acirramento da crise fiscal, quando então passam a ser estimulados o autofinanciamento e o uso de 
fontes de crédito provenientes do setor privado (Coelho, 1998). De qualquer modo, esse retorno aos financiamentos, observado na segunda metade dessa década, não é captado pela análise feita, que apenas permite observar as diferenças nos fatores de modernização entre 1985 e 1995, dada a inexistência de informações relativas ao ano de 1990.

Portanto, observa-se uma contemporaneidade entre os movimentos do fator 1 e do nível de financiamentos tomados pela agricultura, e que está associado com a redução na oferta de crédito rural e elevação do custo de sua obtenção pelos agricultores. Ainda que essa hipótese não seja testada neste artigo, e fuja aos seus objetivos, ela é bastante plausível. Isso porque, mesmo que o volume de financiamentos já constitua, por si só, parte do fator 1, ele não é a única variável a defini-lo. Assim, conquanto não se possa estabelecer uma relação de causalidade, a redução dos valores do fator 1, após 1980, implica a redução não só dos níveis de financiamento, mas de todas as variáveis que compõem este fator, estando a ele fortemente associadas, como é o caso dos indicadores de investimento.

Por outro lado, é importante observar que, mesmo após a cessação desse processo de modernização baseado na oferta abundante de crédito, o movimento de elevação do fator 2, associado ao maior uso de tratores, de força mecânica e de energia elétrica, e à elevação das despesas com a produção, persiste em todo o período restante. O que esses resultados parecem apontar é que certamente ocorreu um processo de redução dos financiamentos tomados pela agricultura, bem como do nível de investimentos feitos na atividade, possivelmente implicando descapitalização dos agricultores, sem que isso tenha acarretado redução no valor dos demais indicadores de modernização, mas certamente provocando um retardo no avanço de algumas de suas variáveis.

Portanto, independentemente da exaustão do principal instrumento de indução da modernização agrícola, o crédito rural subsidiado, esse processo se mostra persistente e irreversível. Com seu avanço, a agricultura se transforma, perdendo a auto-suficiência de suas unidades produtivas, do que resulta o aumento das despesas incorridas na produção, relativas à compra dos diversos insumos que, de forma crescente, se fazem necessários à condução do cultivo.

\section{Conclusões}

Neste artigo, procurou-se analisar o processo modernização agrícola ocorrido em cada unidade da Federação, buscando quantificá-lo e caracterizar sua evolução no período de 1970 a 1995.

Esta análise permitiu concluir que houve diferenças marcantes entre as uni- 
dades da Federação, no que concerne à intensidade com que o processo de modernização agrícola avançou. Nesse sentido, observou-se a modernização agrícola foi mais lenta no grupo formado por Acre, Amapá, Amazonas, Pará, Piauí e Rondônia. No grupo constituído por Bahia, Ceará, Maranhão, Paraíba, Pernambuco, Rio Grande do Norte, Roraima e Sergipe já se observa maior intensidade de modernização do que a constatada para os estados do grupo anterior, sendo, porém, bem inferior ao que se verifica nos demais estados. Um terceiro grupo, composto por Alagoas, Espírito Santo, Goiás, Mato Grosso, Minas Gerais e Rio de Janeiro, define regiões onde o avanço da modernização, embora com diferenças entre estados, é nitidamente superior ao que ocorre nos dois primeiros grupos. Finalmente, Distrito Federal, Mato Grosso do Sul, Paraná, Rio Grande do Sul, Santa Catarina e São Paulo pertencem ao último grupo, que abriga as unidades da Federação onde a modernização agrícola foi a mais intensa no período.

Embora o fenômeno da modernização tenha-se processado com diferentes magnitudes entre as unidades da Federação, sua evolução seguiu um padrão semelhante entre as mesmas.

Basicamente, o traço marcante dessa evolução é a ocorrência de uma inflexão no comportamento dos indicadores relativos ao uso de financiamentos e ao volume de investimentos na agricultura que, até 1980, experimentaram crescimento em praticamente todos os estados. A partir de 1980, observou-se um declínio desses indicadores, movimento que perdurou até o final do período, indicando uma tendência de descapitalização dos agricultores. O declínio desses indicadores está provavelmente relacionado à queda na oferta de crédito rural e à elevação de seu custo, ao que se soma o efeito da retração do mercado, da redução das expectativas e do aumento de riscos observados na década de 1980.

Não obstante a redução desses indicadores, a análise mostrou que as variáveis relativas ao uso de tratores e de fontes não-tradicionais de energia, bem como às despesas com a produção, mantiveram-se em crescimento acelerado após 1980, podendo-se ainda inferir que os demais indicadores tenham experimentado crescimento mais lento nesse período. Isso permite concluir que, apesar da redução na oferta abundante de crédito rural, o processo de modernização agrícola não foi interrompido, muito embora sua evolução, mais lenta a partir de então, denote ainda o predomínio de uma visão de curto prazo, com o declínio nos indicadores de investimento no setor. 


\section{Referências}

Aguirre, B. M. B. \& Bacha, C. J. C. (1989). A especialização da mão-de-obra rural no Brasil. In Congresso Brasileiro de Economia e Sociologia Rural, $2 \%$. Anais..., pages 572-84. Piracicaba.

Buainain, A. M. (1997). Trajetória recente da política agrícola brasileira. Campinas. (Projeto UTF/FAO/036/BRA.).

Campos, J. R. S., Lemos, J. J. S., \& Silva, L. M. R. (1988). Progresso tecnológico e alocação de recursos na agricultura brasileira. Revista de Economia e Sociologia Rural, 26(2):195-212.

Cardoso, J. L. (1992). Estrutura produtiva do setor rural ao nível de unidades da federação. In Congresso Brasileiro de Economia e Sociologia Rural, 29. Anais..., pages 63-68. Brasília, Sober.

Coelho, C. N. (1998). O princípio do desenvolvimento sustentado na agricultura brasileira. Revista de Política Agrícola, 7(2):7-16.

Cunha, M. S. (1995). Modernização da agropecuária no norte paranaense, 197085. In Congresso Brasileiro de Economia e Sociologia Rural, 33. Anais..., pages 713-28. Brasília, Sober.

Duran, B. S. \& Odell, P. L. (1974). Cluster analysis: A survey. Berlin, SpringVerlag. (Lecture notes in economics and mathematical systems, 100.).

Espírito-Santo, E. N. (1998). Agricultura no estado de Santa Catarina, no período 1920-1985. Estudos Econômicos, 28(3):453-73.

Everitt, B. (1977). Cluster Analysis. Heinemann Educational Books, London.

FGV (2000). Banco de dados áries. Disponível em http://www. fgv.br. Acesso em: jan. 2000.

Fibge (s.d.). Censos agropecuários, 1970, 1975, 1980, 1985, 1995/96. Rio de Janeiro, Fibge.

Figueiredo, N. M. S. \& Hoffmann, R. (1998). A dinâmica da modernização da agricultura em 299 microrregiões homogêneas do Brasil: 1975, 1980 e 1985. In Congresso Brasileiro de Economia e Sociologia Rural, 36. Anais..., pages 43950. Poços de Caldas, Sober. 
Gasques, J. G. \& Conceição, J. C. P. R. (1998). Produtividade da agricultura brasileira. In O Agronegócio Brasileiro: Desafios e Perspectivas, pages 425-38. Aguiar, D. R. D. \& Pinho, J. B. (Eds.), Brasília, Sober.

Gomes, M. F. M. (1990). Efeitos da expansão da produção de soja em duas regiões do Brasil. (Tese de Doutorado.).

Hair, J. F., Anderson, R. E., Tatham, R. L., \& Black, W. C. (1995). Multivariate Data Analysis: With Readings. Prentice Hall, New Jersey.

Harman, H. H. (1960). Modern Factor Analysis. University of Chicago Press, Chicago.

Hoffmann, R. (1992). A dinâmica da modernização da agricultura em 157 microrregiões homogêneas do Brasil. Revista de Economia e Sociologia Rural, 30(4):271-90.

Kim, J. \& Mueller, C. W. (1978). Introduction to Factor Analysis: What It is and How to Do It. Sage Publications, London.

Manoel, A. \& Barros, J. R. M. (1987). Agricultura brasileira: Transformações e perspectivas na década de 80. In Encontro Nacional de Economia, 15. Anais..., pages 103-28. Rio de Janeiro, Anpec.

Martine, G. (1989). Modernização e emprego rural no pós-guerra. In Congresso Brasileiro de Economia e Sociologia Rural, 33. Anais..., pages 162-89. Piracicaba, Sober.

Martine, G. \& Beskow, P. R. (1987). O modelo, os instrumentos e as transformações na estrutura de produção agrícola. In Os Impactos Sociais Da Modernização Agrícola, pages 19-39. Martine, G. \& Garcia, R. C. (Orgs.), São Paulo, Caetés.

Meyer, L. F. F. (1997). Modernização da agricultura e desenvolvimento sustentado: O caso de Minas Gerais - 1970 a 1985. Dissertação de Mestrado.

Schilderinck, J. H. F. (1970). Factor Analysis Applied to Developed and Developing Countries. Rotterdan University Press, Groningen.

Silva, J. G. D., Kageyama, A. A., Romão, D. A., Wagner Neto, J. A., \& Pinto, L. C. G. (1983). Tecnologia e campesinato: O caso brasileiro. Revista de Economia Política, 3(4). 
Statistical Package Software (SPSS) (1990). SPSS/PC + advanced statistics 4.0. for the IBM PC/XT/AT and PS/2. Chicago.

Tarsitano, M. A. A. (1992). A modernização e a desconcentração da terra da agricultura mato-grossense: 1970/85. Revista de Economia Política, 12(4):2837 . 


\section{Anexo}

Tabela A.1

Valores dos fatores de modernização para o Brasil e as unidades de Federação:

1970, 1975, 1980, 1985 e 1995

\begin{tabular}{|c|c|c|c|}
\hline \multirow{2}{*}{ Ano } & \multirow{2}{*}{ Região } & \multicolumn{2}{|c|}{ Escores fatoriais } \\
\hline & & $F 1$ & F2 \\
\hline \multirow[t]{27}{*}{1970} & Brasil & 0,111 & $-0,441$ \\
\hline & Acre & $-0,708$ & $-0,332$ \\
\hline & Alagoas & $-0,045$ & $-0,574$ \\
\hline & Amapá & $-0,562$ & $-0,441$ \\
\hline & Amazonas & $-0,629$ & $-0,410$ \\
\hline & Bahia & $-0,413$ & $-0,535$ \\
\hline & Ceará & $-0,354$ & $-0,737$ \\
\hline & Distrito Federal & 0,008 & $-0,066$ \\
\hline & Espírito Santo & $-0,351$ & $-0,305$ \\
\hline & Goiás & $-0,226$ & $-0,545$ \\
\hline & Maranhão & $-0,548$ & $-0,537$ \\
\hline & Mato Grosso & $-0,106$ & $-0,806$ \\
\hline & Mato Grosso do Sul & $-0,253$ & $-0,330$ \\
\hline & Minas Gerais & $-0,169$ & $-0,629$ \\
\hline & Pará & $-0,022$ & $-0,908$ \\
\hline & Paraíba & $-0,205$ & $-0,785$ \\
\hline & Paraná & 0,389 & $-0,562$ \\
\hline & Pernambuco & $-0,191$ & $-0,670$ \\
\hline & Piauí & $-0,448$ & $-0,706$ \\
\hline & Rio de Janeiro & $-0,134$ & $-0,284$ \\
\hline & Rio Grande do Norte & $-0,306$ & $-0,762$ \\
\hline & Rio Grande do Sul & 0,585 & $-0,680$ \\
\hline & Rondônia & $-0,493$ & $-0,420$ \\
\hline & Roraima & $-0,515$ & $-0,446$ \\
\hline & Santa Catarina & 0,403 & $-0,861$ \\
\hline & São Paulo & 0,306 & 0,204 \\
\hline & Sergipe & $-0,180$ & $-0,601$ \\
\hline \multirow[t]{23}{*}{1975} & Brasil & 1,086 & $-0,264$ \\
\hline & Acre & $-0,563$ & $-0,375$ \\
\hline & Alagoas & 1,753 & $-1,330$ \\
\hline & Amapá & $-0,527$ & $-0,451$ \\
\hline & Amazonas & $-0,660$ & $-0,329$ \\
\hline & Bahia & 0,037 & $-0,682$ \\
\hline & Ceará & $-0,106$ & $-0,738$ \\
\hline & Distrito Federal & 0,751 & 0,674 \\
\hline & Espírito Santo & 0,565 & $-0,615$ \\
\hline & Goiás & 0,667 & $-0,576$ \\
\hline & Maranhão & $-0,351$ & $-0,568$ \\
\hline & Mato Grosso & 0,910 & $-1,181$ \\
\hline & Mato Grosso do Sul & 0,907 & $-0,178$ \\
\hline & Minas Gerais & 0,416 & $-0,611$ \\
\hline & Pará & $-0,274$ & $-0,525$ \\
\hline & Paraíba & 0,116 & $-0,852$ \\
\hline & Paraná & 2,011 & $-0,414$ \\
\hline & Pernambuco & 0,314 & $-0,752$ \\
\hline & Piauí & 0,030 & $-1,000$ \\
\hline & Rio de Janeiro & 0,677 & $-0,215$ \\
\hline & Rio Grande do Norte & 0,010 & $-0,838$ \\
\hline & Rio Grande do Sul & 1,977 & $-0,519$ \\
\hline & Rondônia & $-0,548$ & $-0,200$ \\
\hline
\end{tabular}




\begin{tabular}{|c|c|c|c|}
\hline \multirow{2}{*}{ Ano } & \multirow[t]{2}{*}{ Região } & \multicolumn{2}{|c|}{ Escores fatoriais } \\
\hline & & $F 1$ & F2 \\
\hline \multirow{55}{*}{1985} & Roraima & 0,094 & $-0,946$ \\
\hline & Santa Catarina & 1,057 & $-0,464$ \\
\hline & São Paulo & 1,264 & 0,902 \\
\hline & Sergipe & 0,575 & $-0,978$ \\
\hline & Brasil & 1,823 & $-0,272$ \\
\hline & Acre & $-0,620$ & $-0,216$ \\
\hline & Alagoas & 1,680 & $-0,884$ \\
\hline & Amapá & 0,404 & $-1,009$ \\
\hline & Amazonas & $-0,335$ & $-0,494$ \\
\hline & Bahia & 0,139 & $-0,573$ \\
\hline & Ceará & 0,285 & $-0,834$ \\
\hline & Distrito Federal & 4,162 & 0,087 \\
\hline & Espírito Santo & 0,853 & $-0,214$ \\
\hline & Goiás & 0,743 & $-0,230$ \\
\hline & Maranhão & $-0,016$ & $-0,759$ \\
\hline & Mato Grosso & 0,764 & $-0,279$ \\
\hline & Mato Grosso do Sul & 1,760 & 0,727 \\
\hline & Minas Gerais & 0,726 & $-0,293$ \\
\hline & Pará & $-0,431$ & $-0,263$ \\
\hline & Paraíba & 0,682 & $-1,012$ \\
\hline & Paraná & 2,966 & $-0,552$ \\
\hline & Pernambuco & 0,932 & $-0,873$ \\
\hline & Piauí & 0,527 & $-1,247$ \\
\hline & Rio de Janeiro & 0,566 & $-0,015$ \\
\hline & Rio Grande do Norte & 0,672 & $-1,081$ \\
\hline & Rio Grande do Sul & 3,091 & $-0,770$ \\
\hline & Rondônia & $-0,022$ & $-0,516$ \\
\hline & Roraima & 0,556 & $-0,868$ \\
\hline & Santa Catarina & 1,997 & $-0,272$ \\
\hline & São Paulo & 3,049 & 0,559 \\
\hline & Sergipe & 0,507 & $-0,656$ \\
\hline & Brasil & 0,053 & 1,274 \\
\hline & Acre & $-0,642$ & $-0,209$ \\
\hline & Alagoas & 0,477 & $-0,137$ \\
\hline & Amapá & 0,319 & $-0,810$ \\
\hline & Amazonas & $-0,631$ & $-0,252$ \\
\hline & Bahia & $-0,340$ & $-0,165$ \\
\hline & Ceará & $-0,302$ & $-0,470$ \\
\hline & Distrito Federal & 1,138 & 2,482 \\
\hline & Espírito Santo & 0,318 & 0,987 \\
\hline & Goiás & 0,055 & 0,525 \\
\hline & Maranhão & $-0,402$ & $-0,429$ \\
\hline & Mato Grosso & 0,448 & 0,501 \\
\hline & Mato Grosso do Sul & 0,021 & 2,273 \\
\hline & Minas Gerais & $-0,397$ & 0,527 \\
\hline & Pará & $-0,217$ & $-0,539$ \\
\hline & Paraíba & $-0,179$ & $-0,452$ \\
\hline & Paraná & 0,770 & 1,368 \\
\hline & Pernambuco & 0,016 & $-0,240$ \\
\hline & Piauí & $-0,520$ & $-0,344$ \\
\hline & Rio de Janeiro & $-0,447$ & 0,711 \\
\hline & Rio Grande do Norte & $-0,243$ & $-0,395$ \\
\hline & Rio Grande do Sul & 0,556 & 1,100 \\
\hline & Rondônia & $-0,599$ & $-0,089$ \\
\hline & Roraima & $-0,348$ & $-0,224$ \\
\hline
\end{tabular}




\begin{tabular}{llrr}
\hline \multirow{2}{*}{ Ano Região } & \multicolumn{2}{c}{ Escores } & fatoriais \\
\cline { 3 - 4 } & & \multicolumn{1}{c}{$F 1$} & \multicolumn{1}{c}{$F 2$} \\
\hline \multirow{4}{*}{1995} & Santa Catarina & $-0,300$ & 1,565 \\
& São Paulo & $-0,042$ & 3,479 \\
Sergipe & $-0,124$ & $-0,193$ \\
& Brasil & $-1,473$ & 2,185 \\
Acre & $-1,005$ & 0,126 \\
Alagoas & $-0,783$ & 0,405 \\
Amapá & $-0,671$ & $-0,006$ \\
Amazonas & $-0,751$ & $-0,233$ \\
Bahia & $-0,833$ & 0,076 \\
Ceará & $-0,833$ & 0,002 \\
Distrito Federal & $-1,463$ & 4,268 \\
Espírito Santo & $-1,497$ & 1,650 \\
Goiás & $-1,421$ & 1,642 \\
Maranhão & $-0,763$ & $-0,207$ \\
Mato Grosso & $-1,448$ & 2,089 \\
Mato Grosso do Sul & $-2,016$ & 3,342 \\
Minas Gerais & $-1,438$ & 1,417 \\
Pará & $-0,860$ & 0,008 \\
Paraíba & $-0,673$ & 0,039 \\
Paraná & $-1,532$ & 2,754 \\
Pernambuco & $-1,054$ & 0,531 \\
Piauí & $-0,541$ & $-0,420$ \\
Rio de Janeiro & $-1,679$ & 1,695 \\
Rio Grande do Norte & $-1,033$ & 0,345 \\
Rio Grande do Sul & $-1,344$ & 2,296 \\
Rondônia & $-1,104$ & 0,382 \\
Roraima & $-0,748$ & $-0,067$ \\
Santa Catarina & $-1,445$ & 2,663 \\
São Paulo & $-2,095$ & 4,333 \\
Sergipe & $-0,926$ & 0,330 \\
\hline & & \\
& & & \\
& & &
\end{tabular}

